Document downloaded from:

http://hdl.handle.net/10251/100424

This paper must be cited as:

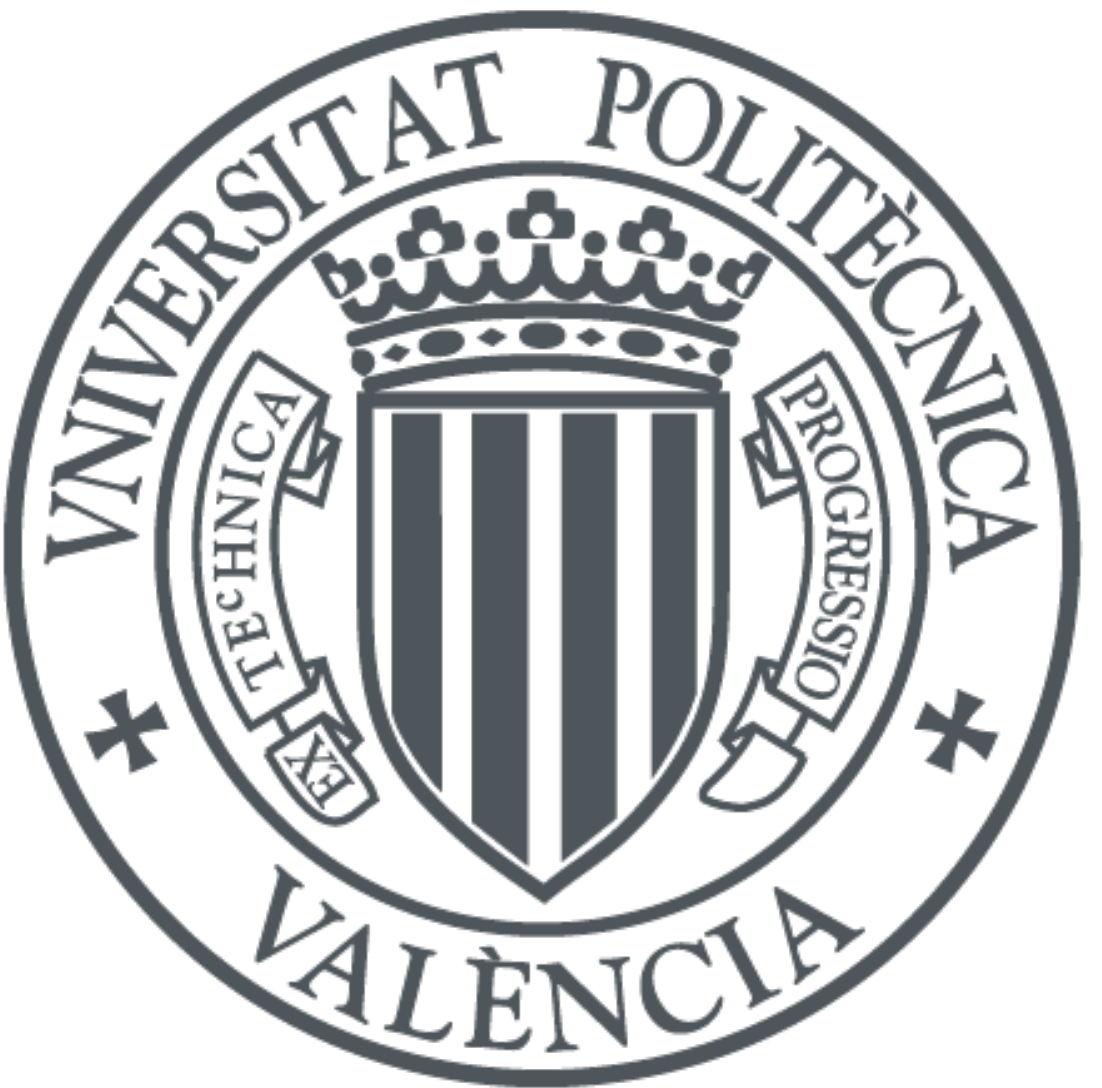

The final publication is available at

https://doi.org/10.1007/PL00011426

Copyright Springer-Verlag

Additional Information 


\title{
A Cutting Plane Algorithm for the General Routing Problem.
}

\author{
Angel Corberán ${ }^{\circ}$ Adam N. Letchford* and José María Sanchis ${ }^{+}$ \\ - DEIO, Faculty of Mathematics, University of Valencia, Spain \\ * Dept. of Management Science, Lancaster University, England \\ + Dept. of Applied Mathematics, University Polytechnic of Valencia, Spain
}

November 9th, 1998.

\begin{abstract}
The General Routing Problem (GRP) is the problem of finding a minimum cost route for a single vehicle, subject to the condition that the vehicle visits certain vertices and edges of a network. It contains the Rural Postman Problem, Chinese Postman Problem and Graphical Travelling Salesman Problem as special cases. We describe a cutting plane algorithm for the GRP based on facet-inducing inequalities and show that it is capable of providing very strong lower bounds and, in most cases, optimal solutions.
\end{abstract}

Key Words: valid inequalities, cutting planes, General Routing Problem, Rural Postman Problem, Graphical Travelling Salesman Problem.

\section{Introduction}

Given a connected, undirected graph $G$ with vertex set $V$ and edge set $E$, a cost $c_{e}$ for each edge $e \in E$, a set $V_{R} \subseteq V$ of required vertices and a set $E_{R} \subseteq E$ of required edges, the General Routing Problem (GRP) is the problem of finding a minimum cost vehicle route passing through each $v \in V_{R}$ and each $e \in E_{R}$ at least once [24].

The GRP is of practical application (see, e.g., [9]) and contains several other important routing problems as special cases:

- When $V_{R}=\emptyset$, the Rural Postman Problem (RPP) is obtained [24]. If, in addition, $E_{R}=E$, the Chinese Postman Problem (CPP) is obtained [8].

- When $E_{R}=\emptyset$, the Steiner Graphical Travelling Salesman Problem (SGTSP) is obtained [7]. This problem was also called the Road Travelling Salesman Problem in [10]. If, in addition, $V_{R}=V$, the Graphical Travelling Salesman Problem (GTSP) is obtained [7].

The RPP was shown to be $\mathcal{N} \mathcal{P}$-hard in [18], although the CPP can be solved in polynomial time by reduction to a matching problem [8]. The GTSP is also $\mathcal{N} \mathcal{P}$-hard [7]. Hence the SGTSP and GRP are also $\mathcal{N} \mathcal{P}$-Hard.

The GTSP is a relaxation of the well-known (Symmetric) Travelling Salesman Problem (STSP): in the GTSP, the route must pass through each vertex at least once and each edge may be traversed any number of times. In the STSP, the route must pass through each vertex 
exactly once and, moreover, $G$ must be a complete graph (see, e.g., $[16,17])$.

In recent years, spectacular progress has been made on solving large-scale STSP instances to optimality. The most successful algorithms to date (e.g., $[2,27]$ ) are based on linear programming relaxations, strengthened by the addition of facet-defining inequalities as cutting planes (see, e.g., [22]). Successful algorithms have also been devised for other routing problems using this paradigm $[2,3,10,11,14,23]$.

An important ingredient of a cutting plane algorithm is the ability to detect inequalities which are violated by the current LP relaxation. For a given class of inequalities, an exact separation algorithm is a routine which takes an LP relaxation as input and outputs one or more violated inequalities in that class (if any exist). A heuristic separation algorithm is similar except that it may fail to detect a violated inequality in the class (see, e.g., [26]).

In [5], various facet-inducing inequalities were discovered for the RPP, including nonnegativity, connectivity, $R$-odd cut and $K$-Component $(K-\mathrm{C})$ inequalities. Using these inequalities, the authors solved 25 out of $26 \mathrm{RPP}$ instances using a pure cutting plane algorithm (the other was solved by branching). However, the violated inequalities were identified by visual inspection rather than by automated separation algorithms.

The present authors have also written three papers on GRP polyhedra. The non-negativity, connectivity, $R$-odd cut and $K$-C inequalities were adapted to the GRP in [6], where also the $K$-C inequalities were generalized to give the honeycomb inequalities. In [19], the path-bridge inequalities were introduced and an exact separation algorithm was proposed for a restricted subclass. Further classes of inequalities were introduced in [20], including projected binested, projected chain and projected bipartition inequalities.

These good theoretical results suggested that it would be possible to solve large-scale GRP instances to optimality, or at least to obtain excellent lower bounds, with a dedicated cutting-plane algorithm. The purpose of the present paper is to give a description of such an algorithm which has been implemented and tested by the authors. This includes a detailed description of some exact and heuristic (polynomial-time) separation algorithms which have been devised for various classes of valid and facet-inducing inequalities.

Note that it can be assumed, without loss of generality, that the end-vertices of any required edge are also required. Our algorithms actually rely on the stronger assumption that $V=V_{R}$. This is not a serious restriction, however, as there is a simple way to transform GRP instances which do not satisfy the assumption into instances which do (see, e.g., [4]). The transformation can occasionally increase the number of edges in $G$, but it frequently decreases it.

In [16], some RPP instances were solved by transforming them into STSP instances and then using a branch-and-cut algorithm for the STSP. Although this worked adequately for the instances tested, the transformation involved the addition of a large number of redundant variables. Our algorithm is more 'natural' and has some dedicated separation algorithms which work very well. We believe that it will be much faster for larger instances.

The outline of the paper is as follows. In Section 2, the relevant results on GRP polyhedra are reviewed. In Section 3, a detailed description is given of the separation algorithms. The way in which these separation algorithms are integrated to form the overall algorithm is described in Section 4. Computational results are given for a wide variety of test problems in Section 5. Some concluding comments are made in Section 6. 


\section{Polyhedral Results}

\section{$2.1 \quad$ Formulation}

The fundamentals of the integer programming approach to the GRP are given in [6]. We use the same notation, with some minor simplifications due to our assumption that $V=V_{R}$. Let $x_{e}$ represent the number of times $e$ is traversed (if $e \notin E_{R}$ ), or one less than this number (if $\left.e \in E_{R}\right)$. Given $S \subset V$, let $\delta(S)$ denote the set of edges, commonly called the edge cutset, with one end-vertex in $S$ and one end-vertex in $V \backslash S$. We also write $E(S: T)$ for $\delta(S) \cap \delta(T)$, $E(S)$ for the set of edges with both end-vertices in $S$ and $x(F)$ for $\sum_{e \in F} x_{e}$. Finally, we write $E_{R}(S: T)$ for $E(S: T) \cap E_{R}$ and $\delta_{R}(S)$ for $\delta(S) \cap E_{R}$. The set of feasible solutions to the GRP is then described by:

$$
\begin{aligned}
x(\delta(S)) \geq 2, & \forall S \subset V, \delta_{R}(S)=\emptyset \\
x(\delta(\{i\})) \equiv \delta_{R}(\{i\}) \bmod 2, & \forall i \in V \\
x \in Z_{+}^{|E|} &
\end{aligned}
$$

The connectivity inequalities (1) ensure that the route is connected. The degree conditions (2) ensure that the vehicle departs each vertex as many times as it arrives. Note that the degree conditions are congruences, not linear equations or inequalities.

The convex hull in $\Re^{|E|}$ of feasible solutions to (1) - (3), known as $\operatorname{GRP}(G)$, is a fulldimensional, unbounded polyhedron. We can therefore formulate the GRP as the problem of minimizing $\sum_{e \in E} c_{e} x_{e}$ subject to $x \in \operatorname{GRP}(G)$. As mentioned in Section 1, many classes of valid inequalities and facets are known for $\operatorname{GRP}(G)[5,6,19,20]$. The most trivial inequalities are the non-negativity inequalities $x_{e} \geq 0$ for each $e \in E$. These induce facets unless $e$ is a cut-edge in $G$.

The non-negativity inequalities are handled implicitly by any LP solver. The authors have devised separation algorithms for five non-trivial classes of inequalities; namely, connectivity, $R$-odd cut, K-C, path-bridge and honeycomb inequalities. These inequalities are described in the following subsections.

\subsection{Connectivity inequalities}

As mentioned in the previous subsection, connectivity inequalities are just constraints (1). They induce facets of $\operatorname{GRP}(G)$ if and only if the subgraphs induced by $S$ and $V \backslash S$ are connected $[5,6]$.

\section{$2.3 \quad R$-odd cut inequalities}

Due to the degree conditions, the vehicle must cross any given edge cutset an even number of times. This means that if $S \subset V$ is such that $\left|\delta_{R}(S)\right|$ is odd, then the $R$-odd cut inequality

$$
x(\delta(S)) \geq 1
$$

is valid for $\operatorname{GRP}(G)$.

Like connectivity inequalities, $R$-odd cut inequalities induce facets of $\operatorname{GRP}(G)$ if and only if the subgraphs induced by $S$ and $V \backslash S$ are connected $[5,6]$. 


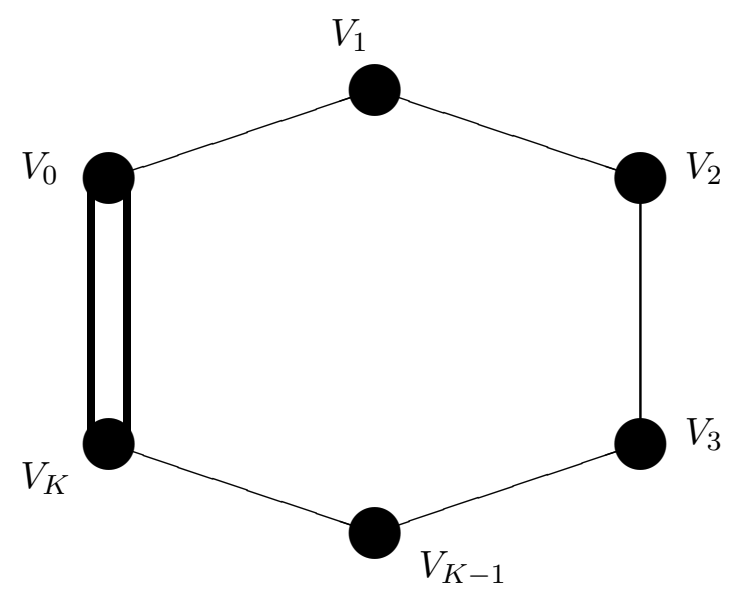

Figure 1: $K$-C configuration.

\section{$2.4 \quad K-\mathrm{C}$ inequalities}

In order to present the remaining inequalities, we will need some more definitions. Consider the (generally disconnected) subgraph of $G$ obtained by deleting all non-required edges from $G$. We call a connected component of this subgraph an $R$-connected component. A set of vertices defining an $R$-connected component will be called an $R$-set. An $R$-set with only one member will be called an isolated vertex.

The $K$-Component or $K-C$ inequalities $[5,6]$ are defined in terms of an associated $K$ $C$ configuration. A $K$-C configuration is (see Figure 1 ) a partition $\left\{V_{0}, \ldots V_{K}\right\}$ of $V$, with $K \geq 3$, such that

- $V_{1}, \ldots V_{K-1}$ and $V_{0} \cup V_{K}$ are clusters of one or more $R$-sets,

- $\left|E_{R}\left(V_{0}: V_{K}\right)\right|$ is positive and even,

- $E\left(V_{i}: V_{i+1}\right) \neq \emptyset$ for $i=0, \ldots, K-1$.

The corresponding $K$-C inequality can be written as:

$$
F(x)=\sum_{i=0}^{K-1} x\left(\delta\left(V_{0} \cup \cdots \cup V_{i}\right)\right)-2 x\left(E\left(V_{0}: V_{K}\right)\right) \geq 2(K-1)
$$

$K$-C inequalities induce facets of $\operatorname{GRP}(G)$ when certain mild connectivity assumptions are met $[5,6]$.

\subsection{Path-bridge inequalities}

The path-bridge (PB) inequalities [19] are defined in terms of an associated path-bridge (PB) configuration. Suppose $p \geq 1$ and $b \geq 0$ are integers such that $p+b \geq 3$ and odd. Let $n_{i} \geq 2$ for $i=1, \ldots, p$ also be integers. A PB configuration is (see Figure 2) a partition of $V$ into vertex sets $A, Z$ and $V_{j}^{i}$ for $i=1, \ldots, p, j=1, \ldots, n_{i}$ with the following properties:

- each $V_{j}^{i}$ is a cluster of one or more $R$-sets, 


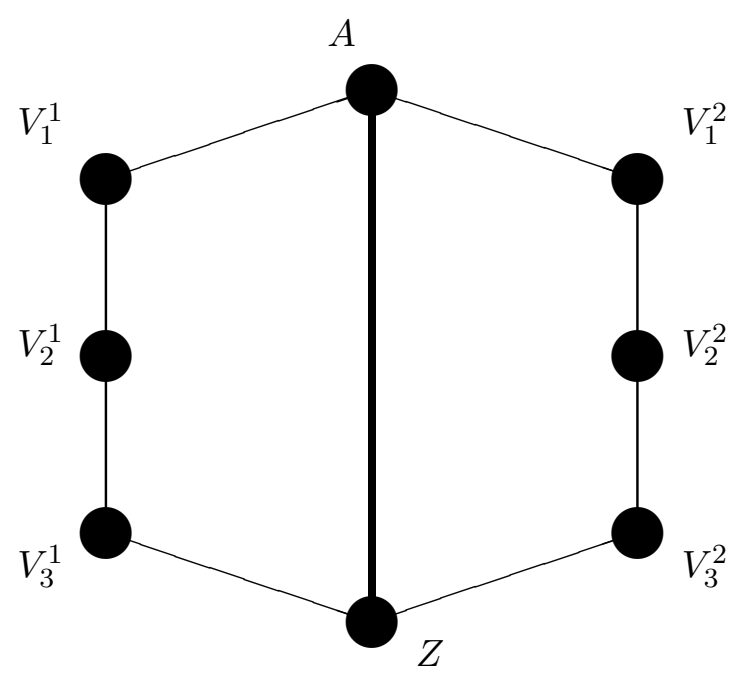

Figure 2: PB configuration.

- $\left|E_{R}(A: Z)\right|=b$,

- $E\left(A: V_{1}^{i}\right) \neq \emptyset$ and $E\left(V_{n_{i}}^{i}: Z\right) \neq \emptyset$ for $i=1, \ldots, p$,

- $E\left(V_{j}^{i}: V_{j+1}^{i}\right) \neq \emptyset$ for $i=1, \ldots, p$ and $j=1, \ldots, n_{i}-1$.

The edges in $E_{R}(A: Z)$ constitute the bridge. When $b=0$, the bridge is empty and the $\mathrm{PB}$ configuration reduces to a path configuration, see [7]. In such a case, either or both of $A$ and $Z$ are permitted to be empty.

The formula for the coefficients in the PB inequality is rather complicated. However, our separation algorithms (Subsections 3.5 and 3.6) are designed for so-called $n$-regular PB inequalities, in which all of the $n_{i}$ are equal to the same value $n[7,19]$. For such inequalities, there is a nice description of the coefficients in terms of vertex sets called handles and teeth. There are $n-1$ handles, denoted by $H_{1}, \ldots, H_{n-1}$, and $p$ teeth, denoted by $T_{1}, \ldots, T_{p}$ (see Figure 3). The first handle is defined as $H_{1}=A \cup V_{1}^{1} \cup \ldots \cup V_{1}^{p}$; the other handles are defined inductively as $H_{i}=H_{i-1} \cup V_{i}^{1} \cup \ldots \cup V_{i}^{p}$. The teeth are defined as $T_{j}=V_{1}^{j} \cup \ldots \cup V_{n}^{j}$. The $n$-regular PB inequality is then:

$$
\sum_{i=1}^{n-1} x\left(\delta\left(H_{i}\right)\right)+\sum_{j=1}^{p} x\left(\delta\left(T_{j}\right)\right) \geq n p+n+p-1
$$

and is valid for $\operatorname{GRP}(G)$. It is facet-inducing under mild connectivity assumptions.

For brevity, we will call regular $\mathrm{PB}$ inequalities $n-P B$ inequalities (where $n$ may or may not be specified). Certain special cases of $n$-PB inequalities are of note. When $p=1$, a $K-\mathrm{C}$ inequality is obtained. When $b=0$, a regular path inequality is obtained [7]. The 2-PB inequalities are analogous to the well-known comb inequalities for the STSP (see, e.g., [13]). Finally, a 2-PB inequality in which each tooth consists merely of two isolated vertices (connected by a non-required edge) is called simple [19]. Simple 2-PB inequalities are analogous to the 2-matching inequalities for the STSP [13]. 


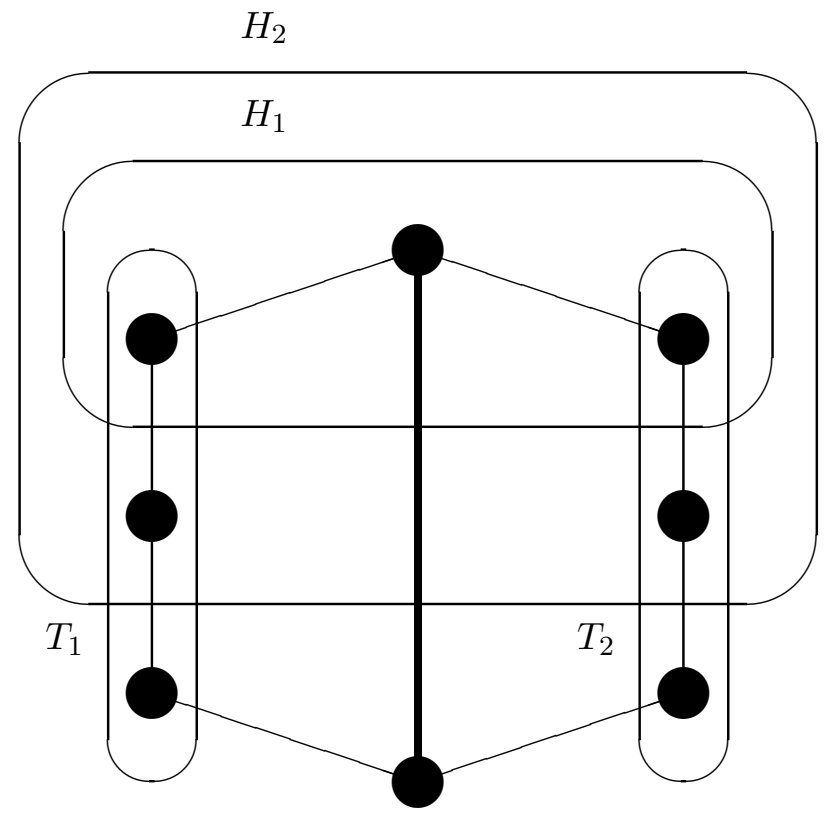

Figure 3: Handles and teeth in a 3-regular PB configuration.

\subsection{Honeycomb inequalities}

The honeycomb inequalities [6], like PB inequalities, are also a generalization of $K$-C inequalities. However, the generalization is in a different direction and neither class contains the other. A honeycomb configuration is a partition of $V$ into sets $S_{i}$ such that:

- for all $i,\left|\delta\left(S_{i}\right) \backslash \delta_{R}\left(S_{i}\right)\right| \neq \emptyset$ and $\left|\delta_{R}\left(S_{i}\right)\right|$ is even or zero;

- there are at least two values $i$ such that $\delta_{R}\left(S_{i}\right) \neq \emptyset$;

- there are at least two values $i$ such that $\delta_{R}\left(S_{i}\right)=\emptyset$;

together with a set of non-required edges crossing between the $S_{i}$ which form a tree spanning the $S_{i}$.

Many, but not all, honeycomb configurations can be formed by 'gluing' $K$-C configurations together by identifying edges [6]. In general, honeycomb configurations can be extremely complicated and computing the coefficients in the associated honeycomb inequality can be a formidable task. For this reason, we restrict ourselves in this paper to honeycomb configurations with a special structure. These consist of:

- a partition $\left\{V_{1}, \ldots, V_{L}, W_{1}, \ldots, W_{K-1}\right\}$ of $V$, with $L \geq 2, K \geq 3$, such that $\left(V_{1} \cup \ldots \cup\right.$ $\left.V_{L}\right), W_{1}, \ldots, W_{K-1}$ are clusters of one or more $R$-sets, $\delta\left(V_{i}\right)$ contains a positive and even number of required edges for all $i$ and the graph induced by the required edges on the vertex set $\left\{V_{1}, \ldots, V_{L}\right\}$ is connected.

- a tree $T$ spanning the sets $V_{1}, \ldots, V_{L}, W_{1}, \ldots, W_{K-1}$ such that the degree in $T$ of every vertex set $V_{i}$ is 1 , the degree of vertex sets $W_{j}$ is at least 2 and the path in the tree connecting any distinct $V_{i}, V_{j}$ is of length 3 or more. 

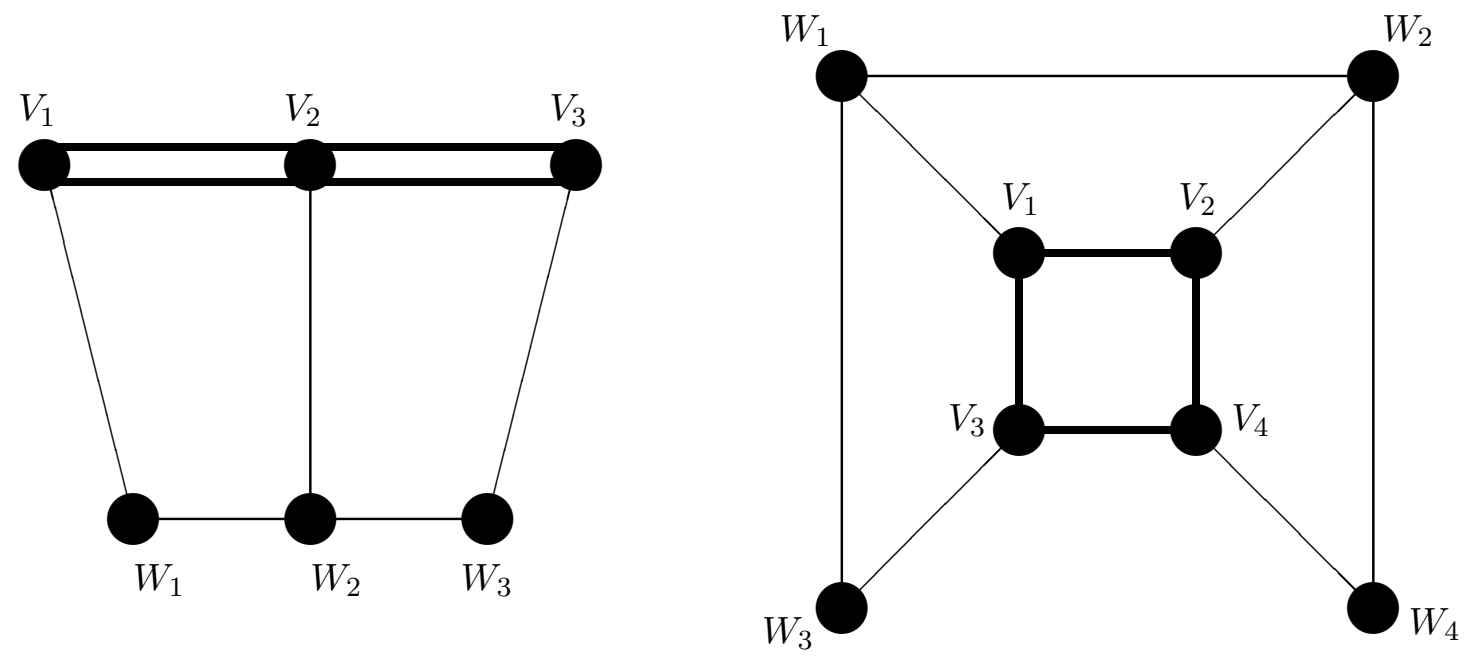

Figure 4: Two suitable honeycomb configurations.

If $L=2$ the tree degenerates to a mere path and we have a $K$-C configuration. If $L \geq 3$, then $K \geq 4$ is needed in order for the path in the tree connecting any distinct $V_{i}, V_{j}$ to be of length 3 or more.

For honeycomb configurations with this special structure, the coefficient $\alpha_{e}$ of edge $e \in E$ in the associated honeycomb inequality is equal to the number of edges traversed (if any) in the spanning tree to get from one end-vertex of $e$ to the other, except for the edges with one end-vertex in $V_{i}$ and the other in $V_{j}, i \neq j$, when the coefficient is 2 units less. The honeycomb inequality is then:

$$
\sum_{e \in E} \alpha_{e} x_{e} \geq 2(K-1)
$$

These honeycomb inequalities define facets of $\operatorname{GRP}(G)$ if certain mild connectivity assumptions are met.

Figure 4 shows two suitable honeycomb configurations. The bold lines represent edges in $\delta_{R}\left(V_{i}\right)$ for some $i$ and the thin lines represent edges in the spanning tree. The rhs of the associated inequality is 6 for the configuration on the left and 8 for the configuration on the right.

\section{Separation Algorithms}

\subsection{Preliminary Comments}

In this section, separation algorithms are presented for the inequalities described in the previous section. Before proceeding, however, we will need some definitions.

Given an LP relaxation vector $x^{*} \in \Re_{+}^{|E|}$, define the weighted graph $G_{*}\left(V, E, x^{*}\right)$. This weighted graph is input to all of the separation procedures. Given a graph $G(V, E)$, a cutvertex is a vertex the removal of which causes $G$ to become disconnected. A block is a maximal connected subgraph of $G$ which contains no cut-vertices. If a block consists of a single edge, then it is called a cut-edge. A decomposition of $G$ into blocks can be found in 
$\mathcal{O}(|E|)$ time using the algorithm in [29]. We will also need the concept of shrinking a set of vertices in a weighted graph (see, e.g., $[10,26])$. Given a graph $G(V, E)$ with weights on the edges, and a set $W \subset V$, shrinking $W$ means identifying the vertices in $W$, deleting any resulting loops and merging each resulting set of parallel edges, if any, into a single edge. When merging parallel edges, the new edge is given a weight equal to the sum of the original weights. Shrinking can be done iteratively and, in the case of the GRP, a single edge in the shrunk graph can represent a mixture of any number of required or non-required edges in the original graph.

In the following subsections, we describe heuristic (and sometimes exact) separation algorithms for each of the classes of inequalities described in the previous section. Some of the heuristics require the choice of a parameter (called $\epsilon$ ), or even two parameters (called $\epsilon_{1}, \epsilon_{2}$ ). For details on which values of $\epsilon$ (or $\epsilon_{1}, \epsilon_{2}$ ) we use and how the exact and heuristic algorithms are embedded in the overall algorithm, see Subsection 4.2.

\subsection{Connectivity Separation}

Connectivity inequalities can be separated exactly in polynomial time. This is done by finding a minimum weight cut in the shrunk graph $G_{s}=\left(V_{s}, E_{s}, \bar{x}^{*}\right)$ obtained from $G_{*}$ by shrinking each $R$-set into a single vertex. A minimum weight cut in an undirected graph with $n$ vertices and $m$ edges can be found in $\mathcal{O}\left(n m+n^{2} \log n\right)$ time using the algorithm in [21].

A faster $(\mathcal{O}(|E|)$ time $)$ heuristic algorithm is obtained by computing the connected components of the subgraph induced by the edges $e \in E_{s}$ with $\bar{x}_{e}^{*}>\epsilon$, where $\epsilon$ is a given parameter. Let $S_{1}, S_{2}, \ldots, S_{q}$ be the sets of vertices in the original graph $G$ corresponding to the vertex sets of these connected components. Then $x\left(\delta\left(S_{i}\right)\right) \geq 2$ is a violated connectivity inequality if $q>1$ and $x^{*}\left(\delta\left(S_{i}\right)\right)<2$. Note that when $q=2$ we have $x\left(\delta\left(S_{1}\right)\right)=x\left(\delta\left(S_{2}\right)\right)$, but when $q>2$ all of the inequalities are distinct.

\section{$3.3 \quad R$-odd cut separation}

$R$-odd cut inequalities can also be separated exactly in polynomial time. Before describing the exact algorithm, however, we describe an effective separation heuristic. As for the connectivity inequalities, we choose a parameter $\epsilon$. We compute the vertex sets $S_{1}, S_{2}, \ldots, S_{q}$ of the connected components of the subgraph of $G_{*}$ induced by the edges $e \in E$ with $x_{e}^{*}>\epsilon$. Then $x\left(\delta\left(S_{i}\right)\right) \geq 1$ is a violated $R$-odd cut inequality if $q>1,\left|\delta_{R}\left(S_{i}\right)\right|$ is odd and $x^{*}\left(\delta\left(S_{i}\right)\right)<1$.

This heuristic runs in $\mathcal{O}(|E|)$ time and is inspired by a heuristic presented in [12] for blossom inequalities in the context of the perfect matching problem.

The exact algorithm is much slower and involves finding a minimum weight $R$-odd cutset in $G_{*}$. Using a result of [25], this can be reduced to a series of maximum flow problems on $G_{*}$. The number of maximum flow computations needed in the worst case is equal to the number of $R$-odd vertices in $G$ minus 1 and an $R$-odd cut inequality is violated if and only if the weight of the cutset is less than 1 .

As noted in [26] (in the context of the STSP), the idea of connected components can also be used to simplify the problem of finding an odd cut of weight less than 1 . Consider the connected components of the subgraph of $G_{*}$ induced by the edges $e \in E$ with $x_{e}^{*}>0$. Under the assumption that the separation heuristic has already been (unsuccessfully) invoked with $\epsilon=0$, we know that each of these components contains an even number of $R$-odd vertices. It is easy to show that we can examine each of these components independently for an odd cut of weight less than 1 . This typically leads to considerable speed improvements in the exact algorithm. 


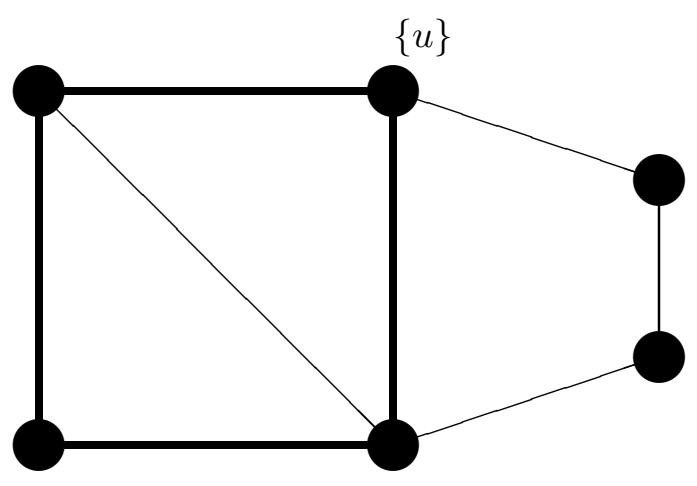

Figure 5: Getting seeds for $V_{0}$ and $V_{K}$.

\section{$3.4 \quad K$-C separation}

A vertex $v \in V$ will be called $R$-odd if $\left|\delta_{R}(\{v\})\right|$ is odd, otherwise it will be called $R$-even. Isolated vertices are $R$-even.

It is not known if the problem of separating $K-\mathrm{C}$ inequalities can be solved in polynomial time or not, but our guess is that this problem is $\mathcal{N} \mathcal{P}$-hard. However, we have designed a heuristic algorithm which appears to work very well. It has three consecutive phases. In phase I, we find 'seeds' for $V_{0}$ and $V_{K}$. In phase II, we use these seeds to determine the $V_{i}$ for $i=0, \ldots, K$. In phase III, we check the resulting inequality for violation, and, if it is not violated, we also check some other inequalities for violation which are obtained by shrinking the $K$-C configuration appropriately.

The details of these three phases are based on the following considerations:

- We have examined the structure of the solutions obtained when all the connectivity and $R$-odd cut inequalities are satisfied. The effect of the $K$-C inequalities is to separate solutions $x^{*}$ where an $R$-even vertex $u$ belonging to an $R$-set $C_{i}$ with $\left|C_{i}\right|>2$ satisfies $x^{*}(\delta(\{u\})) \cong 1$ and $x^{*}\left(E\left(\{u\}: C_{i} \backslash\{u\}\right)\right) \cong 0$. Thus, $\{u\}$ and $C_{i} \backslash\{u\}$ are suitable vertex sets to be considered as seeds for $V_{0}$ and $V_{K}$, respectively, in phase I. Figure 5 illustrates this idea: the bold lines represent the required edges in $E\left(C_{i}\right)$ and the narrow lines represent non-required edges with $x_{e}^{*}=1$. This idea is generalized by considering as seeds for $V_{0}$ any vertex sets with an even number of $R$-odd vertices forming an $x^{*}$-connected component on the subgraph induced by $C_{i}$.

- Given the seeds for $V_{0}$ and $V_{K}$, consider the graph obtained from $G_{*}$ by shrinking the seeds and the remaining $R$-sets into a single vertex each. In order to define $V_{0}, \ldots, V_{K}$, we have to find a path in the shrunk graph connecting the seeds (preferably one with a large $x^{*}$-weight). Once this is done, we merely have to assign any vertices which are not in the path to one of the vertices in the path in order to complete phase II.

- Suppose that a $K$-C inequality with $K \geq 4$ is not violated. For $i=0,1,2, \ldots, K-1$, let $L H S(i)=x^{*}\left(\delta\left(V_{0} \cup \ldots \cup V_{i}\right)\right)$. For some $1 \leq i \leq K-2$, we could merge sets $V_{i}, V_{i+1}$ into a single set, yielding a new 'smaller' $(K-1)$-C configuration, with an associated $(K-1)-\mathrm{C}$ inequality. From equation (5), for a given $x^{*}$, the slack of the new inequality will be equal to that of the original inequality, plus $2-L H S(i)$. Thus, iteratively merging sets $V_{i}, V_{i+1}$ with $L H S(i)>2$ will lead to inequalities with smaller slack, as long as $K \geq 3$ remains. 
Alternatively, consider what would happen if we were to merge sets $V_{0}, \ldots, V_{s}$ into a single set for some $s \leq K-3$. The resulting change in slack would be $2 s-\sum_{i=1}^{s}(L H S(i-$ 1) $\left.+2 x^{*}\left(V_{i}: V_{K}\right)\right)$. Then, if this quantity was negative, the slack would have been reduced by shrinking. A similar argument applies if we merge sets $V_{t}, \ldots, V_{K}$ for some $t \geq 3$.

It can also be shown that a $K$-C inequality cannot be violated if $x^{*}\left(E\left(V_{0}: V_{K}\right)\right) \geq 1$ and $x^{*}$ satisfies all connectivity and R-odd cut inequalities.

These are the ideas behind phase III.

In our computer code, a given $K$-C configuration is stored by simply labelling each vertex in the graph according to the set $V_{i}$ of the $K$-C configuration it belongs to. Computing coefficients from these labels is a simple matter and so is the operation of merging two or more $V_{i}$ into one.

Taking into account the above points, the global procedure is as follows:

- Phase I: Define seeds for $V_{0}$ and $V_{K}$.

Given a specified $R$-set $C_{i}$, we say that a vertex in $C_{i}$ is $x^{*}$-external if it is connected to at least one vertex in a different $R$-set by an edge $e$ with $x_{e}^{*}>0$. For each $R$-set $C_{i}$ with at least two $x^{*}$-external vertices and connected to at least two different $R$-sets, do the following. Choose a parameter $\epsilon$ and construct the subgraph of $G\left(C_{i}\right)$ induced by edges $e$ with $x_{e}^{*}>\epsilon$. Look for an $x^{*}$-external vertex $u$ whose corresponding connected component in the subgraph has an even number of $R$-odd vertices. If one is found, the connected component is a candidate for $V_{0}$ and the complementary set in $C_{i}$ is a candidate for $V_{K}$ (if $V_{K} \neq \emptyset$ ).

- Phase II: Define the vertex sets $V_{0}, \ldots, V_{K}$.

Given the seeds for $V_{0}$ and $V_{K}$, we proceed as follows:

(a) construct the graph obtained from $G_{*}$ by shrinking the seeds and the remaining $R$-sets into a single vertex each.

(b) Compute a spanning tree by iteratively adding the edge of maximum weight not forming a cycle (and not connecting the seeds). If this is not possible (because the removal of $C_{i}$ disconnects the graph), then move on to another $x^{*}$-external vertex.

(c) Transform the tree into a path linking the seeds, by (iteratively) shrinking each non-seed vertex with degree one into its (unique) adjacent vertex. If the length of the path is only 2 , then move on to another $x^{*}$-external vertex. Otherwise, the vertices of the path define the vertex sets $V_{0}, V_{1}, \ldots, V_{K}$. Note that the seeds may have been enlarged in this process and therefore that either or both of $V_{0}$ and $V_{K}$ may contain vertices from more than one $R$-set.

\section{- Phase III: Check the $K-\mathrm{C}$ inequality.}

We now have a $K$-C configuration like that of Figure 1.

For each $i=0,1, \ldots, K-1$, compute $L H S(i)=x^{*}\left(\delta\left(V_{0} \cup \ldots \cup V_{i}\right)\right)$. Then, $F\left(x^{*}\right)=$ $\sum_{i=0}^{K-1} L H S(i)-2 x^{*}\left(V_{0}, V_{K}\right)$ is the left hand side of the corresponding $K$-C inequality computed on $x^{*}$. If $F\left(x^{*}\right)<2(K-1)$, the $K$-C inequality is violated.

If the $K$-C inequality is not violated, check if a violated inequality could be obtained by shrinking the $K$-C configuration.

- Shrink all pairs $V_{i}, V_{i+1}$ with $L H S(i)>2,1 \leq i \leq K-2$ and reduce $K$ accordingly. If $K<3$, stop (no violated inequality was found). 
- Compute the values:

$$
\begin{aligned}
& a=\operatorname{LHS}(0)+2 x^{*}\left(E\left(V_{1}: V_{K}\right)\right)-2 \\
& b=\operatorname{LHS}(K-1)+2 x^{*}\left(E\left(V_{0}: V_{K-1}\right)\right)-2 \\
& c=a+b+2 x^{*}\left(E\left(V_{1}: V_{K-1}\right)\right)
\end{aligned}
$$

corresponding to the reductions in slack which would be obtained if we shrank, respectively, the pair $V_{0}, V_{1}$ or the pair $V_{K-1}, V_{K}$, or both pairs simultaneously. If $\max \{a, b, c\}$ is larger than the slack, then shrink the pair $V_{0}, V_{1}$ and/or the pair $V_{K-1}, V_{K}$ (depending on where the maximum of $\{a, b, c\}$ is reached), to obtain a violated $K$-C inequality (if $K \geq 3$ remains).

- If this fails, it may yet be possible to obtain a violated inequality by shrinking. Compute for every $0 \leq s, t \leq K-3, s+t \leq K-3$, the reduction in slack (denoted by $A_{s t}$ ) which would be obtained if we shrank sets $V_{0}, V_{1}, \ldots V_{s}$ and sets $V_{K}, V_{K-1} \ldots V_{K-t}$ into single sets (this can be done quickly if the computations are performed in an intelligent way). If $A_{s t}>$ slack for any $s, t$ such that $K \geq 3$ would remain after shrinking, then select the $s, t$ which gives the inequality with greatest violation.

For a fixed value of $\epsilon$, the algorithm looks for only one violated $K$-C inequality associated to each $R$-set. For each $R$-set $C_{i}$, priority is given to single vertices in $C_{i}$ as candidates for $V_{0}$. Only if this fails to yield a violated inequality for the given $C_{i}$ are more complex candidates for $V_{0}$ considered.

A simple extension of the heuristic allows us to search for violated $K$-C inequalities where the required edges in $E\left(V_{0}: V_{K}\right)$ belong to more than one $R$-connected component. This is done by (iteratively) joining a pair of $R$-sets adjacent in $G_{*}$ and then applying the above procedure as if they formed a single $R$-set.

\section{5 $n$-PB separation: the case $n=2$}

When considering the separation of $n$-PB inequalities, it is useful to give the 2-PB inequalities special attention. Accordingly, the present subsection is concerned with 2-PB inequalities, whereas general $n$-PB inequalities are dealt with in the following subsection.

In [19] it was shown, using ideas in [25], that the simple 2-PB inequalities can be separated exactly in polynomial time, provided that two conditions are met:

- $x^{*}$ satisfies all non-negativity and $R$-odd cut inequalities,

- $x^{*}(\delta(e))+x_{e}^{*} \geq 3$ holds for each $e \in E$.

The first condition is obviously no problem. As for the second condition, it is easy to show that it is satisfied whenever $x^{*}$ satisfies all connectivity inequalities.

There are however two problems with the simple 2-PB separation algorithm. First, it is rather slow as it involves the computation of a minimum weight odd cut in a graph with $\mathcal{O}(|E|)$ vertices and $\mathcal{O}(|E|)$ edges. This problem can be alleviated to some extent by using the idea of connected components, as outlined in Subsection 3.3. The second problem is that there are likely to be very few violated simple 2-PB inequalities, if any, when the GRP instance has few isolated vertices. Indeed, simple 2-PB inequalities are not defined at all for RPP instances.

One way of improving the simple 2-PB separation algorithm would be to find conditions under which $G_{*}$ can be shrunk (see Subsection 3.1) in such a way that 
- A 2-PB inequality is violated in the shrunk graph if and only if a 2-PB inequality is violated by $x^{*}$,

- A violated simple 2-PB inequality in the shrunk graph can be 'expanded' into a (not necessarily simple) 2-PB inequality violated by $x^{*}$.

Although this idea has been applied successfully to related separation problems (see $[10,26]$ ), we did not use it here because it turned out to be very difficult to devise appropriate data structures to account for the presence of required edges. A different idea was used instead.

Consider the graph $G_{*}^{\prime}$ obtained from $G_{*}$ by deleting non-required edges $e$ with $x_{e}^{*}=0$. Suppose we have a block decomposition of $G_{*}^{\prime}$ (see Subsection 3.1) and that there are $h \geq 2$ blocks. Let $V(j)$ for $j=1, \ldots, h$ denote the set of vertices in block $j$, let $G^{j}$ for $j=1, \ldots, h$ denote the subgraph of $G_{*}^{\prime}$ induced by $V(j)$ and let $x(j)$ denote the vector obtained from $x^{*}$ by dropping all components apart from those corresponding to edges in $E(V(j))$. It is possible to show that a 2-PB inequality is violated by $x^{*}$ if and only if there is at least one $j$ such that a 2-PB inequality (valid for $\operatorname{GRP}\left(G^{j}\right)$ ) is violated by $x(j)$.

We therefore apply the simple 2-PB algorithm to each $G^{j}$ separately. If a violated simple 2-PB inequality is found for some $G^{j}$, we expand it into a (not necessarily simple) 2-PB inequality violated by $x^{*}$. This is done by viewing $G^{j}$ as being obtained from $G_{*}^{\prime}$ by shrinking, in any order, those $V(i)$ such that $i \neq j$. This approach typically yields considerable speed improvements, because it is frequently the case that there are $h \geq 2$ blocks. Moreover, it typically leads to more violated inequalities being discovered. Even for RPP instances, where there are no isolated required vertices in the original graph $G$, it is possible for one or more blocks to contain isolated required vertices and therefore for violated simple 2-PB inequalities to be found on individual blocks.

To avoid difficulties due to rounding errors, we actually define $G_{*}^{\prime}$ a little differently: we delete from $G_{*}$ all non-required edges $e$ with $x_{e}^{*}<0.01$.

\section{$3.6 n$-PB separation: the general case}

The separation of $n$-PB inequalities appears to be very difficult and is likely to be $\mathcal{N} \mathcal{P}$-hard. However, the authors have devised a separation heuristic which is fast (it can be implemented to run in $\mathcal{O}(|V| .|E| . \log |V|)$ time) and quite effective. In fact, it has proved worthwhile to run this heuristic before the heuristic for 2-PB inequalities described in the previous subsection.

Like the 2-PB heuristic, the $n$-PB heuristic is applied to each block $G^{j}$ of $G_{*}$ separately and each resulting violated inequality is 'expanded' to yield an inequality violated by $x^{*}$. From now on we assume that we are working on an individual block, but for simplicity of notation we assume that there is in fact only one block, the graph $G_{*}$ itself.

Given any two $R$-sets $S_{1}, S_{2}$ connected by at least one edge, let $\theta\left(S_{1}, S_{2}\right)=x^{*}\left(\delta\left(S_{1} \cup\right.\right.$ $\left.\left.S_{2}\right)\right)+x^{*}\left(E\left(S_{1}: S_{2}\right)\right)-3$. In limited experiments, adjacent pairs $V_{j}^{i}$ and $V_{j+1}^{i}$ in violated $n$-PB inequalities often turned out to be $R$-sets $S_{1}, S_{2}$ such that $\theta\left(S_{1}, S_{2}\right)$ was small. This fact is used in the first stage of the heuristic, which is to get a good set $\mathcal{H}$ of candidates for $H_{1}$ :

- Choose two parameters $0 \leq \epsilon_{1}, \epsilon_{2} \leq 1$.

- Initially all edges are unlabelled. 
- Examine each pair $S_{1}, S_{2}$ of $R$-sets connected by at least one edge. If $\theta\left(S_{1}, S_{2}\right) \leq \epsilon_{1}$, then label the edges in $E\left(S_{1}\right)$ and $E\left(S_{2}\right)$ 'strong' and the edges in $E\left(S_{1}: S_{2}\right)$ 'weak'. Store $\left(S_{1}, S_{2}\right)$ as a 'candidate tooth'. $S_{1}$ and $S_{2}$ are the 'ends' of the candidate tooth.

- Examine the remaining unlabelled edges. Label such an edge $e$ 'weak' if $x_{e}^{*} \leq \epsilon_{2}$.

- Delete all weak edges from $G_{*}$.

- Examine each connected component in the resulting graph. Let $C$ be the set of vertices in one such component. Let $b=\left|\delta_{R}(C)\right|$ and let $p$ equal the number of candidate teeth with exactly one end in $C$. If $p+b \geq 3$ and odd, $p \geq 1$ and the $p$ candidate teeth mentioned are vertex-disjoint, then put $C$ into the list $\mathcal{H}$ of candidates for $H_{1}$.

It is possible to implement this to run in $\mathcal{O}(|E|)$ time, by first of all computing $x^{*}(\delta(S))$ for each $R$-set $S$. Notice also that, for fixed $\epsilon_{1}, \epsilon_{2},|\mathcal{H}|$ is $\mathcal{O}(|V|)$. The remainder of the heuristic is repeated for each candidate $H_{1} \in \mathcal{H}$.

First, we check whether the simple 2-PB inequality with handle $H_{1}$ and the $p$ candidate teeth is violated. If so, we store this violated inequality. Whether or not this simple 2-PB inequality is violated, we then proceed to look for a more general $n$-PB inequality which is violated.

To this end, we set $V_{1}^{1}, \ldots, V_{1}^{p}$ to be the ends of the $p$ candidate teeth lying in $H_{1}$. The remainder of $H_{1}$, if any, is used to form $A$. The other ends of the $p$ candidate teeth are used to form 'seeds' for $V_{2}^{1}, \ldots, V_{2}^{p}$. We then construct a shrunk graph from $G_{*}$ as follows:

- Shrink the $R$-sets which are contained entirely in $H_{1}$ into a single vertex each, and do the same for the $R$-sets which are contained entirely in $V \backslash H_{1}$.

- Shrink the vertices in $H_{1}$ which are incident on one or more crossing required edges into a single 'inner pole' vertex (unless there are no such vertices).

- Shrink the vertices in $V \backslash H_{1}$ which are incident on one or more crossing required edges into a single 'outer pole' vertex (unless there are no such vertices).

Let $G_{s}\left(V_{s}, E_{s}, \bar{x}^{*}\right)$ denote the weighted shrunk graph and let $H_{s}$ denote the shrunk $H_{1}$. In the next stage, $G_{s}$ is used to enlarge the $\mathrm{PB}$ configuration. We distinguish two cases.

- Case 1: $b \geq 1$. Here, $A$ is non-empty and we also have an obvious 'seed' for $Z$; namely, the outer pole. If $\bar{x}^{*}(E(A: Z)) \geq 1$, we give up. Otherwise we follow a similar idea to that used in the $K$-C heuristic: we iteratively add edges in order of non-increasing weight so as to build a tree in $G_{s}$ with the following properties:

- It spans all vertices in $V_{s}$ apart from those representing $A$ and the $V_{1}^{i}$.

- It consists of $p+1$ edge-disjoint subtrees.

- Subtree $i$ for $i=1, \ldots, p$ contains a path from the $V_{2}^{i}$ seed to the $Z$ seed but does not contain any paths from the $V_{2}^{i}$ seed to any other $V_{2}^{j}$ seed, $j \neq i$.

- Subtree $p+1$ (which may be empty) connects the $Z$ seed to other vertices, but does not contain any paths from $Z$ to any of the $V_{2}^{i}$ seeds.

Once the structure has been made, we iteratively shrink vertices of degree one (different from the $V_{2}^{i}$ and $Z$ seeds) into their unique adjacent vertex to obtain a (not necessarily regular) $\mathrm{PB}$ configuration. 
- Case 2: $b=0$. Here, $p \geq 3$, it is possible for $A$ to be empty and we do not have a 'seed' for $Z$. We build two structures, one in which $Z$ is forced to be empty and one in which $Z$ is forced to non-empty.

In the first case, we build a forest in $G_{s}$ which spans all vertices in $V_{s}$ apart from those representing $A$ and the $V_{1}^{i}$. It must consist of $p$ vertex-disjoint trees, such that each of the $V_{2}^{i}$ seeds is contained in exactly 1 tree. Once the forest is made, we pick longest paths going out from each of the $V_{2}^{i}$ seeds to get the $p$ necessary paths and shrink vertices of degree 1 as before.

In the second case, we build a tree in $G_{s}$ which spans all vertices in $V_{s}$ apart from those representing $A$ and the $V_{1}^{i}$. Now, an edge is permitted to create a path connecting two distinct $V_{2}^{i}$ seeds. Once this happens, one of the vertices on the path (different from the $V_{2}^{i}$ seeds) must now be a $Z$ seed. The remainder of the tree is built with this in mind: The addition of an edge forming a path connecting one of the possible $Z$ seeds to a third $V_{2}^{i}$ seed is not permitted unless it is incident on a potential $Z$ seed (which is then chosen as the actual $Z$ seed). If this never happens (because the shrunk graph is not sufficiently connected), the tree is abandoned. Otherwise, we proceed as in case 1 now that we have our $Z$ seed.

At this stage, we will generally have a non-regular PB configuration (or possibly two). Moreover, the structure of the configuration in the vicinity of $Z$ is likely to be 'messy'. We deal with both of these problems simultaneously: We choose $n$ according to the length of the shortest of the $p$ paths in the configuration and shrink any paths which are longer than this by merging $Z$ with adjacent $V_{j}^{i}$.

Now that we have an $n$-PB inequality, we check if is violated. If not, we may still be able to obtain a violated inequality by 'removing handles' (analogous to shrinking a $K$-C configuration). It is easy to see from the inequality (6) that the slack of the $n$-PB inequality will be decreased by removing any handle $H_{i}$ such that $x^{*}\left(\delta\left(H_{i}\right)\right)>p+1$ and merging adjacent $V_{j}^{i}$ accordingly. This is done iteratively, as long as $n \geq 2$ remains.

\subsection{Honeycomb separation}

As for the $K$-C inequalities, we first try to separate honeycomb inequalities in which a single $R$-connected component is partitioned into more than 2 parts. The effect of these honeycomb inequalities is to separate solutions $x^{*}$ where some $R$-even vertices $u_{1}, u_{2}, \ldots, u_{L}$ (or sets with an even number of $R$-odd vertices) belonging to an $R$-set $C_{i}$ satisfy $x^{*}\left(\delta\left(u_{j}\right)\right) \cong 1$ and $x^{*}\left(E\left(\left\{u_{j}\right\}: C_{i} \backslash\left\{u_{j}\right\}\right)\right) \cong 0, j=1,2, \ldots, L$.

The idea of our algorithm is, given an $R$-connected component, to find the $x^{*}$-external vertices, say $u_{1}, u_{2}, \ldots, u_{t}$, and, then, assign the remaining vertices to some of the previous ones in order to define vertex sets $V_{1}, V_{2}, \ldots, V_{t}$ in such a way that $x^{*}\left(E\left(V_{i}: V_{j}\right)\right)$ is as small as possible, for $1 \leq i<j \leq t$. The procedure is as follows:

Phase I: Define seeds for $V_{1}, V_{2}, \ldots, V_{L}$.

Given an $R$-set $C_{i}$, we assign to each $x^{*}$-external vertex $u_{j}$ a label $k$ corresponding to the $R$-set $C^{k}$ with $x^{*}\left(\left\{u_{j}\right\}: C^{k}\right), k \neq i$ maximum. To each of the remaining vertices in $C_{i}$, a different negative label is assigned. Starting with the edge $(u, v)$ in $E\left(C_{i}\right)$ with largest $x^{*}$-weight, let $l_{\min }\left(l_{\max }\right)$ be the smaller (larger) label of that of $u$ and $v$. We assign the label $l_{\max }$ to all vertices in $C_{i}$ having label $l_{\min }$. This procedure is repeated until all vertices 
have positive label. Vertices with the same label define a partition $V_{1}, V_{2}, \ldots, V_{t}$ of the set of vertices of $C_{i}$. If the number of $R$-odd vertices in each $V_{j}, j=1, \ldots, t$ is even, we are done. Otherwise, all the sets $V_{j}$ with an odd number of $R$-odd vertices are joined, forming a single set. Hence, we have defined a partition $V_{1}, V_{2}, \ldots, V_{L}$. If $L \geq 3$, these sets are suitable to be considered as part of a honeycomb configuration. If $L=2$, these sets are suitable to be considered as $V_{0}$ and $V_{K}$ for a $K$-C configuration. Since this way of defining $V_{0}$ and $V_{K}$ is different from that of Phase I in the above $K$-C separation algorithm, it is worthwhile to look for a violated $K$-C inequality. We then apply Phases II and III of $K$-C separation. Otherwise $(L=1)$, we study another $R$-connected component.

Phase II: Define the vertex sets $W_{1}, W_{2}, \ldots, W_{K-1}$ and the tree.

After (seeds for) $V_{1}, V_{2}, \ldots, V_{L}$ have been defined, we consider the graph obtained by shrinking each $V_{1}, V_{2}, \ldots, V_{L}$ and each of the remaining $R$-sets into single vertices. As in the separation of $K$-C and $n$-PB inequalities, we try to compute a spanning tree with large $x^{*}$ weight in this shrunk graph, by iteratively adding the edge of maximum weight not forming a cycle (and not crossing between the $V_{i}$ ). Initially we forbid adding edges of zero weight. If we obtain a tree without having to add an edge of zero weight, we then shrink (iteratively) each vertex with degree one on the tree (different from $V_{1}, V_{2}, \ldots, V_{L}$ ) into its (unique) adjacent vertex, thus obtaining the vertex sets $W_{1}, W_{2}, \ldots, W_{M}$ and the tree defining the honeycomb configuration. If we do not, then before adding edges of zero weight, we check each one of the connected components in the forest to see whether it defines a honeycomb configuration by itself. Only if this also fails do we add to the forest the edges of zero weight needed to get a spanning tree. If the degree of any vertex $V_{i}$ on the tree is more than two, we reject that honeycomb configuration and consider another $R$-connected component. Otherwise, for each vertex $V_{i}$ incident with two vertices, $W_{j}$ and $W_{k}$, on the tree, we replace one of the edges $\left(V_{i}, W_{j}\right)$ or $\left(V_{i}, W_{k}\right)$ (that with largest $x^{*}$-weight) by edge $\left(W_{j}, W_{k}\right)$ on the tree. Then, every vertex $V_{i}$ has degree one in the configuration tree.

If the above procedure has been successful, we now have a honeycomb configuration defined by $V_{1}, V_{2}, \ldots, V_{L}$ and $W_{1}, W_{2}, \ldots, W_{K-1}$ and its corresponding inequality $F(x) \geq$ $2(K-1)$.

\section{Phase III: Check the Honeycomb inequality.}

For a given vector $x^{*}$, in order to compute $F\left(x^{*}\right)$, we define a vector with a record $T(e)$ for each edge in the configuration tree. For each edge $e \in E$, we add $x_{e}^{*}$ to the records corresponding to the edges in the (unique) path on the tree joining the two 'shrunk' vertices containing the two end-vertices of edge $e$. Then, we can compute easily

$$
F\left(x^{*}\right)=\sum_{e \in T} T(e)-2 \sum_{1<i<j<L} x^{*}\left(V_{i}: V_{j}\right)
$$

Given a honeycomb configuration, if we shrink any pair of sets $W_{i}, W_{j}$ adjacent in the tree into a single one, we will obtain a new 'smaller' honeycomb inequality. Furthermore, when we shrink $W_{i}, W_{j}$, the obtained inequality will be of the form $F^{\prime}(x) \geq 2(K-2)$, where $F^{\prime}\left(x^{*}\right)=F\left(x^{*}\right)-T(i, j)$. Hence, shrinking sets $W_{i}, W_{j}$ with $T\left(\left(W_{i}, W_{j}\right)\right)>2$ leads to honeycomb inequalities with greater violation. As for the $K-\mathrm{C}$ inequalities, if a given honeycomb inequality is not violated, we check if we will obtain a new violated honeycomb inequality by shrinking pairs of adjacent vertices on the tree and if so we shrink them accordingly. We do not shrink sets of the form $V_{i}, W_{j}$, since doing this could lead to a honeycomb configuration in which a vertex of type $V_{j}$ has degree more than one in the tree, a situation which was 
forbidden in Subsection 2.6 to make the computation of coefficients tractable.

As for the separation of $K$-C inequalities (Subsection 3.4), the above procedure can also be extended to search for violated honeycomb inequalities in which the required edges crossing between $V_{i}$ and $V_{j}$ belong to more than one $R$-connected component.

\section{The Overall Algorithm}

In this section we will describe how the separation algorithms are integrated to form the overall cutting-plane algorithm.

\subsection{Initial Relaxation}

The first stage in the algorithm is to construct an initial LP relaxation to be input to the LP solver. We have found that a good choice is to include one connectivity inequality for each $R$-component and one $R$-odd cut inequality for each $R$-odd vertex (together with the non-negativity inequalities, which are handled implicitly by the LP-solver). We also found it useful to include the upper bounds $x_{e} \leq 2$ for each $e \in E$. (It is easy to show that these upper bounds hold in any optimal solution).

We also want to mention that, for some problems, it is possible to fix some variables at zero. If, for three vertices $i, j, k$, the cost of edge $\{i, j\}$ is equal to the sum of the costs of edges $\{i, k\}$ and $\{k, j\}$, then edge $\{i, j\}$ is redundant and its associated variable can be removed from the problem. However, this criterion only applied to a few of our test instances (see Section 5).

\subsection{Inequalities Added in Each Iteration}

In each iteration of the cutting-plane algorithm, the exact and heuristic separation algorithms are invoked in a specific order and a number of violated inequalities are added to the LP relaxation, which is then resolved using the dual simplex method. We have experimented with various priority orderings for the separation algorithms. The following ordering works very well for problems in which $E_{R} \neq \emptyset$ :

1. $R$-odd cut and connectivity separation heuristics with $\epsilon=0$.

2. If no violated inequality was found in step 1 , then repeat with $\epsilon=0.25$.

3. If no violated inequality was found in step 2 , then repeat with $\epsilon=0.5$.

4. Exact connectivity separation if the heuristics failed.

5 . Exact $R$-odd cut separation if the heuristics failed.

6. If the number of violated inequalities detected so far is $>10$, stop.

7. $K$-C separation heuristic with $\epsilon=0$.

8. If no violated inequality was found in step 7 , then repeat with $\epsilon=0.25$.

9. If no violated inequality was found in step 8, then repeat with $\epsilon=0.5$.

10. If the number of violated inequalities detected so far is $>15$, stop. Else do honeycomb separation heuristic (that can also find $K$-Cs).

11. If the number of violated inequalities detected so far is $>15$, stop. Else do $n$-PB heuristic with $\epsilon_{1}=0,0.25,0.5,0.75$ and 1 and $\epsilon_{2}=\min \left\{0.25, \epsilon_{1}\right\}$.

12. If the number of violated inequalities detected so far is $>5$, stop. Else do heuristics for $K$-Cs and honeycombs with iterative merging of adjacent $R$-components.

13. If no violated inequality has been found so far, then do 2-PB heuristic (which is exact 
for simple 2-PBs).

When $E_{R}=\emptyset$ (i.e., when the GRP instance is a SGTSP instance), it is better to use the following strategy:

1. Connectivity separation heuristics with $\epsilon=0$.

2. If no violated inequality was found in step 1 , then repeat with $\epsilon=0.25$.

3. If no violated inequality was found in step 2 , then repeat with $\epsilon=0.5$.

4. If the number of violated inequalities detected so far is $>15$, stop. Else do $n$-PB heuristic with $\epsilon_{1}=0,0.25,0.5,0.75$ and 1 and $\epsilon_{2}=\min \left\{0.25, \epsilon_{1}\right\}$.

5. If no violated inequalities have been detected so far (or, every fifth iteration, no connectivity inequalities have been found so far), do exact connectivity separation algorithm.

6. If no violated inequality has been found so far, then do 2-PB heuristic.

\subsection{Constraint Management and the Cut Pool}

When a large number of cuts have been added, the LPs can become rather large, causing speed and memory problems. To alleviate this problem, it is helpful to occasionally 'purge' constraints from the LP formulation. We found that a good strategy was to remove, every 10 iterations, any constraints which have a slack of 0.01 or more.

However, it can happen that a constraint which was removed at one time can become violated in a subsequent iteration. Because of the heuristic nature of some of the separation algorithms, this could lead to a useful constraint being lost forever. To avoid this difficulty, we use the idea of a cut pool [27]. Every time a violated inequality is found, it is stored in the cut pool. Then, whenever the LP is purged, the cut pool is checked to see if it contains any violated constraints. If so, these are added to the LP and the LP is then re-solved.

We also search through the cut pool whenever the separation algorithms fail to find a violated inequality.

\subsection{Additional Phase}

If no more violated inequalities can be found and the solution vector $x^{*}$ still does not represent an optimal tour, we do some more things.

First, we use a recent result in [11]. Consider the graph obtained by shrinking each $R$-set of $G$ into a single vertex, but without merging parallel edges. Construct a minimum cost spanning tree $T$ on this graph (using the $c_{e}$ as costs). Then there is an optimal solution to the GRP such that the following conditions hold:

- $x_{e} \leq 2$ for all $e \in T$,

- $x_{e} \leq 1$ for all $e \notin T$.

These upper bounds dominate the ones mentioned in Subsection 4.1. We insert these upper bounds into the LP relaxation and then call the separation procedures again. (The reason that we do not put these upper bounds in from the very start is that, on some instances, their presence can create spurious 'odd' vertices in the LP relaxation.)

If this still does not yield the optimal tour and the lower bound is not an integer value, we append an extra inequality which enforces integrality of the objective and then call the separation procedures again. (For example, if the lower bound was 100.23, then we would add the constraint $\sum_{e \in E} c_{e} x_{e} \geq 101$.) 
If, after all this, the LP relaxation is still not integral, we invoke branch-and-bound. To avoid the solution of very large integer programs (IPs), we first delete all constraints with a slack of 0.01 or more. We have found that the resulting IP is usually solved quickly and often leads to the optimal solution of the GRP. However, it is possible that the IP solution might not be feasible for the GRP, since the associated 'tour' could fail to be connected, Eulerian, or both. In such a case, the procedure terminates with a (very tight) lower bound, but no feasible GRP solution.

\section{Computational Experiments}

The algorithm has been coded in the $C$ language and run on a SUN Sparc 20 workstation, using the CPLEX library to solve the LP problems. (Note that the SUN machine is rather slow. Its speed is comparable to a $66 \mathrm{MhZ}$ Pentium). The algorithm has been tested on RPP and GTSP instances, as well as 'genuine' GRP instances. A description of the test instances follows:

- 24 RPP instances, denoted by I1 to I24, from [4] and two more RPP instances, denoted by IAA and IAB, from [5] obtained by randomly selecting some edges in the real-world based graph, with 116 vertices and 174 edges, of the city of Albaida (Valencia) (Table 1).

- 92 RPP instances from [15]. In these problems, the vertices lie on the Euclidean plane and edge costs are proportional to Euclidean distances. The set $E$ is formed according to one of three procedures and then the set $E_{R}$ is chosen randomly. In the first procedure (used for 20 instances, Table 2), $E$ is made of edges with short length in the plane. In the second procedure, (used for 36 instances, Table 3), $E$ forms a unit grid graph. In the third procedure (also used for 36 instances, Table 4), $G$ is a graph in which all vertices have degree 4 , made from two edge-disjoint Hamiltonian cycles found by a nearest neighbour heuristic. These instances have $16 \leq|V| \leq 100$ and $24 \leq|E| \leq 200$.

- 10 GRP instances generated from the Albaida graph by visually selecting the required edges. We select, for example, edges corresponding to streets oriented from north to south, from east to west, forming cycles, etc. (Table 5).

- 15 GRP instances randomly generated from the Albaida graph, by defining each edge as required with probability $P$ and considering all the vertices of the graph as required. Five graphs were generated for each value of $P=0.7,0.5$ and 0.3 , named alb-7-1 to alb-3-5 (Table 6).

- 15 GRP instances, named mad-7-1 to mad-3-5, randomly generated from the real-world based graph of the city of Madrigueras (Albacete) — with 196 vertices and 316 edges — in the same way as for Albaida (Table 7).

- 7 GTSP instances with between 150 and 225 vertices and between 296 and 433 edges (Table 8). These were formed by taking planar Euclidean TSP instances from TSPLIB [28] and making the associated graphs sparse by deleting all edges apart from

- The edges in an optimal TSP tour

- The edges connecting each vertex to its three nearest neighbours. 
For these instances, the cost of the optimal solution was known beforehand. Also, some of the edges in these instances could be eliminated using the criterion given in Subsection 4.1. However, we only did this for the two largest instances (rat195g and ts225g).

In the following tables, LB0 shows the lower bound values corresponding to the initial relaxations, while LB shows the lower bound values obtained at the end of the cutting-plane procedure. For the cases where branch and bound was used, the solution cost is given in the B\&B column. Total running times have also been included. For each instance in Tables 1 and 5 to 8 , the number of identified violated connectivity, $R$-odd cut, $K$-C, honeycomb, $K$-C and honeycombs with merging of adjacent $R$-sets, $n$-PB and simple 2 -PB constraints are presented under headings conn, odd, $\mathrm{K}-\mathrm{C}, \mathrm{HC}, \mathrm{KC} 2, \mathrm{HC} 2, \mathrm{nPB}$ and $\mathrm{SPB}$, respectively.

In all tables, $\mathrm{a}^{\text {'*' }}$ on a $\mathrm{LB}$ or $\mathrm{B} \& \mathrm{~B}$ entry means that not only the optimal value but also an optimal GRP solution was obtained using the cutting-plane algorithm alone, or the cutting-plane algorithm plus $\mathrm{B} \& \mathrm{~B}$, respectively. $\mathrm{A}^{(*)}$ on an UB entry means that this upper bound is optimal.

In Tables 2 to 4 the entries corresponding to the different types of constraints and to the running time represent the accumulated amount for all the instances in each group. Moreover, the number of instances in each group solved to optimality with cutting-planes alone and the number of instances that were solved to optimality after B\&B are shown.

Finally, the upper bounds shown in Table 5 for instances grp4 and grp10 were obtained by Alain Hertz (private communication) using the methods in [15]. Instances grp4 and grp10 were built to be difficult, but it is our belief that both upper bounds should be optimal or very near optimal, as the sophisticated heuristic procedures of [15] produced optimal solutions for all the instances reported in Tables 1 to 4 and for the 8 other instances of Table 5 .

It will be seen that 116 out of 118 RPP instances were solved to optimality by cutting planes alone and the other 2 were solved after invoking branch and bound. The maximum percentage gap between the LP lower bound value and the optimal cost was $0.26 \%$ for these instances. Thus, the algorithm works extremely well for RPP instances.

When it comes to 'genuine' GRP instances, it will be seen that 28 out of 40 instances were solved to optimality by cutting planes alone and a further 6 were solved by branch and bound. The maximum percentage gaps between the LP lower bound and the optimal cost or upper bound were $1.81 \%$ for instance grp10 in Table 5, 0.5\% for instance alb-3-1 in Table 6 and $0.37 \%$ for instance mad-5-5 in Table 7 .

Finally, 3 out of the 7 GTSP instances, 6 if we resorted to branch and bound, were solved to optimality. The worst performance was obtained for the instance ts225g. For this instance, the percentage gap was $1.62 \%$, and the branch-and-bound algorithm failed to find the optimal integer solution after 20,000 nodes had been explored. (Note, however, that the corresponding TSPLIB instance, ts225, was deliberately constructed to be difficult for cutting plane algorithms). It will also be noticed that the running times were somewhat larger for the GTSP instances than for the RPP and GRP instances. Even so, they are acceptable given that our machine is at least 8 times slower than a modern PC.

\section{Conclusions}

The computational results given in the previous section show that our cutting plane algorithm is capable of solving many GRP instances of realistic size to proven optimality. This provides 
further evidence, if evidence were needed, that the study of integer polyhedra is important from a practical as well as a theoretical point of view.

Of course, there is still room for improvement in our algorithm. One obvious improvement would be the addition of better separation algorithms. In this context, 'better' could simply mean faster, or it could mean generating more inequalities or inequalities of greater generality. Another improvement would be to embed the separation algorithms within a full branch-andcut scheme (see [27]).

Finally, we would also like to mention that the separation procedures presented in this paper could be useful for more complex routing problems, perhaps containing multiple vehicles, directed arcs or other complications encountered in practice (see [9] for a survey).

Acknowledgements: The authors want to thank Alain Hertz for providing us his instances and upper bounds.

\section{References}

[1] D. Applegate, R.E. Bixby, V. Chvátal \& W. Cook (1995) Finding cuts in the TSP. DIMACS Technical Report TR 95-05, Rutgers University, New Brunswick, NJ, USA.

[2] P. Augerat, J.M. Belenguer, E. Benavent, A. Corberán, D. Naddef \& G. Rinaldi (1995). Computational results with a branch-and-cut code for the capacitated vehicle routing problem. Research report RR949-M, ARTEMIS-IMAG, France.

[3] J.M. Belenguer \& E. Benavent (1998) The capacitated arc routing problem: valid inequalities and facets. Computational Optimization 83 Applications, 10, 165-187.

[4] N. Christofides, V. Campos, A. Corberán \& E. Mota (1981) An algorithm for the rural postman problem. Imperial College Report IC.OR.81.5. London.

[5] A. Corberán \& J.M. Sanchis (1994) A polyhedral approach to the rural postman problem. Eur. J. Opl Res., 79, 95-114.

[6] A. Corberán \& J.M. Sanchis (1998) The general routing problem polyhedron: facets from the RPP and GTSP polyhedra. Eur. J. Opl Res., 108, 538-550.

[7] G. Cornuéjols, J. Fonlupt \& D. Naddef (1985) The travelling salesman on a graph and some related integer polyhedra. Math. Prog., 33, 1-27.

[8] J. Edmonds \& E.L. Johnson (1973) Matchings, Euler tours and the Chinese postman problem. Math. Prog., 5, 88-124.

[9] H.A. Eiselt, M. Gendreau \& G. Laporte (1995) Arc-routing problems, part 2: the rural postman problem. Oper. Res., 43, 399-414.

[10] B. Fleischmann (1985) A cutting-plane procedure for the travelling salesman problem on a road network. Eur. J. Opl Res., 21, 307-317.

[11] G. Ghiani \& G. Laporte (1997) A branch-and-cut algorithm for the undirected rural postman problem. Working paper, Centre for Research on Transportation, University of Montréal. 
[12] M. Grötschel \& O. Holland (1985) Solving matching problems with linear programming. Math. Prog., 33, 243-259.

[13] M. Grötschel \& M.W. Padberg (1979) On the symmetric travelling salesman problem I: inequalities. Math. Prog., 16, 265-280.

[14] M. Grötschel \& Z. Win (1992) A cutting plane algorithm for the windy postman problem. Math. Prog., 55, 339-358.

[15] A. Hertz, G. Laporte \& P. Nanchen (1998) Improvement procedures for the undirected rural postman problem. Working paper, Centre for Research on Transportation, University of Montréal.

[16] M. Jünger, G. Reinelt \& G. Rinaldi (1995) The travelling salesman problem. In Ball et al. (Eds.) Network Models. Handbooks on Operations Research and Management Science Vol. 7. Amsterdam: Elsevier.

[17] M. Jünger, G. Reinelt \& G. Rinaldi (1997) The travelling salesman problem. In Dell'Amico et al. (Eds.) Annotated Bibliographies in Combinatorial Optimization. New York: Wiley.

[18] J.K. Lenstra \& A.H.G. Rinnooy-Kan (1976) On general routing problems. Networks, 6, $273-280$

[19] A.N. Letchford (1997a) New inequalities for the general routing problem. Eur. J. Opl Res., 96, 317-322.

[20] A.N. Letchford (1997b) The general routing polyhedron: a unifying framework. To appear in Eur. J. Opl Res., Vol. 112 (1999).

[21] H. Nagamochi, T. Ono \& T. Ibaraki (1994) Implementing an efficient minimum capacity cut algorithm. Math. Prog., 67, 325-341.

[22] G.L. Nemhauser \& L.A. Wolsey (1988) Integer and Combinatorial Optimization. New York: Wiley.

[23] Y. Nobert \& J.-C. Picard (1996) An optimal algorithm for the mixed Chinese postman problem. Networks, 27, 95-108.

[24] C.S. Orloff (1974) A fundamental problem in vehicle routing. Networks, 4, 35-64.

[25] M.W. Padberg \& M.R. Rao (1982) Odd minimum cut-sets and b-matchings. Math. Oper. Res., 7, 67-80.

[26] M.W. Padberg \& G. Rinaldi (1990) Facet identification for the symmetric travelling salesman polytope. Math. Prog., 47, 219-257.

[27] M.W. Padberg \& G. Rinaldi (1991) A branch-and-cut algorithm for the resolution of large-scale symmetric travelling salesman problems. SIAM Rev., 33, 60-100.

[28] G. Reinelt (1991) TSPLIB - a travelling salesman problem library. ORSA J. on Comput., 3, 376-384.

[29] R.E. Tarjan (1972) Depth first search and linear graph algorithms. SIAM J. on Comp., 1, 146-60. 


\begin{tabular}{|c|c|c|c|c|c|c|c|c|c|c|c|c|}
\hline & \multirow[b]{2}{*}{$|V|$} & \multirow{2}{*}{$\begin{array}{c}\mathrm{R}- \\
\text { sets }\end{array}$} & \multirow[b]{2}{*}{ LB0 } & \multicolumn{7}{|c|}{ constraints } & \multirow[b]{2}{*}{$\mathrm{LB}$} & \multirow[b]{2}{*}{$\begin{array}{c}\text { time } \\
\text { (scs.) }\end{array}$} \\
\hline & & & & conn. & odd & $\mathrm{K}-\mathrm{C}$ & $\mathrm{KC} 2$ & $\mathrm{HC}$ & $\mathrm{HC} 2$ & $\mathrm{nPB}$ & & \\
\hline I01 & 11 & 4 & 31 & 5 & 10 & - & - & - & - & - & $51^{*}$ & 0.03 \\
\hline I02 & 14 & 4 & 62 & 4 & 10 & 4 & - & - & - & - & $72^{*}$ & 0.04 \\
\hline I03 & 28 & 4 & 24 & 5 & 16 & 9 & 4 & - & - & - & $29^{*}$ & 0.10 \\
\hline I04 & 17 & 3 & 24 & 3 & 14 & 7 & - & - & - & - & $29^{*}$ & 0.10 \\
\hline $\mathrm{I} 05$ & 20 & 5 & 49 & 5 & 14 & 14 & 9 & - & - & - & $55^{*}$ & 0.17 \\
\hline I06 & 24 & 7 & 27 & 8 & 24 & 13 & 6 & - & - & - & $32^{*}$ & 0.23 \\
\hline I07 & 23 & 3 & 35 & 3 & 18 & - & - & - & - & - & $37^{*}$ & 0.08 \\
\hline I08 & 17 & 2 & 26.5 & 2 & 16 & - & - & - & - & - & $29 *$ & 0.09 \\
\hline I09 & 14 & 3 & 18 & 3 & 12 & 1 & - & - & - & - & $26^{*}$ & 0.06 \\
\hline $\mathrm{I} 10$ & 12 & 4 & 27 & 5 & 10 & 8 & - & - & - & - & $35^{*}$ & 0.10 \\
\hline I11 & 9 & 3 & 9 & 3 & 6 & - & - & - & - & - & $9 *$ & 0.08 \\
\hline $\mathrm{I} 12$ & 7 & 3 & 6 & 3 & 6 & 2 & - & - & - & - & $6 *$ & 0.02 \\
\hline $\mathrm{I} 13$ & 7 & 3 & 23 & 3 & 6 & - & - & - & - & - & $23^{*}$ & 0.01 \\
\hline $\mathrm{I} 14$ & 28 & 6 & 43 & 9 & 28 & 18 & 6 & - & - & - & $57^{*}$ & 0.32 \\
\hline $\mathrm{I} 15$ & 26 & 8 & 207 & 16 & 30 & 2 & 1 & - & - & - & $261^{*}$ & 0.23 \\
\hline I16 & 31 & 7 & 53 & 8 & 25 & 14 & 3 & 6 & - & 2 & $64^{*}$ & 0.64 \\
\hline $\mathrm{I} 17$ & 19 & 5 & 39 & 7 & 16 & 9 & 6 & 2 & 1 & - & $49^{*}$ & 0.17 \\
\hline $\mathrm{I} 18$ & 23 & 8 & 54 & 12 & 26 & - & - & 1 & 1 & - & $85^{*}$ & 0.17 \\
\hline I19 & 33 & 7 & 112 & 7 & 30 & 3 & - & - & - & - & $116^{*}$ & 0.12 \\
\hline $\mathrm{I} 20$ & 50 & 7 & 80 & 12 & 34 & 26 & 8 & 3 & 2 & - & $116^{*}$ & 0.54 \\
\hline $\mathrm{I} 21$ & 49 & 6 & 52 & 9 & 40 & 41 & 19 & 8 & 3 & - & $78 *$ & 1.25 \\
\hline $\mathrm{I} 22$ & 50 & 6 & 103 & 7 & 30 & 3 & - & 1 & - & - & $122^{*}$ & 0.31 \\
\hline I 23 & 50 & 6 & 78 & 10 & 45 & 51 & 32 & 2 & 2 & 2 & $95 *$ & 1.18 \\
\hline I 24 & 41 & 7 & 96 & 8 & 33 & 28 & 16 & 6 & 5 & 2 & $113^{*}$ & 1.17 \\
\hline IAA & 102 & 10 & 2896 & 11 & 114 & 105 & 90 & 15 & 15 & 3 & $3304^{*}$ & 6.98 \\
\hline IAB & 90 & 11 & 1728 & 17 & 87 & 11 & 4 & 4 & 2 & - & $2826^{*}$ & 2.42 \\
\hline
\end{tabular}

Table 1: Computational results for the Christofides et al (1981) and Corberán \& Sanchis (1994) instances. 


\begin{tabular}{||c|c|c||c|c|c|c|c|c|c||c|c|c||}
\hline \hline \multirow{2}{*}{$\mathrm{n}$} & \multirow{2}{*}{$\begin{array}{c}\text { \# of } \\
\text { inst. }\end{array}$} & $\begin{array}{c}\text { R-sets } \\
\text { average }\end{array}$ & conn. & odd & K-C & KC2 & HC & HC2 & nPB & $\begin{array}{c}\text { TO of } \\
\text { LB } \\
\text { opt. }\end{array}$ & $\begin{array}{c}\text { \# of } \\
\text { B\&B } \\
\text { opt. }\end{array}$ & $\begin{array}{c}\text { Total time } \\
\text { (scs.) for all } \\
5 \text { instances }\end{array}$ \\
\hline \multirow{2}{*}{20} & 5 & 3.4 & 18 & 44 & 2 & - & - & - & - & 5 & - & 0.20 \\
30 & 5 & 4.8 & 30 & 80 & 24 & 13 & - & - & - & 5 & - & 0.56 \\
40 & 5 & 7.0 & 46 & 107 & 31 & 17 & 2 & 1 & 2 & 5 & - & 1.18 \\
50 & 5 & 8.2 & 59 & 130 & 13 & 10 & 4 & 2 & 4 & 5 & - & 1.60 \\
\hline
\end{tabular}

Table 2: Computational results for Class 1 RPP random instances from Hertz et al. (1998).

\begin{tabular}{|c|c|c|c|c|c|c|c|c|c|c|c|c|c|}
\hline \multirow[b]{2}{*}{$\mathrm{n}$} & \multirow[b]{2}{*}{ Prob. } & \multirow{2}{*}{$\begin{array}{l}\text { \# of } \\
\text { inst. }\end{array}$} & \multirow{2}{*}{$\begin{array}{l}\text { R-sets } \\
\text { average }\end{array}$} & \multicolumn{7}{|c|}{ TOTAL constraints for all 3 instances } & \multirow{2}{*}{$\begin{array}{l}\text { \# of } \\
\text { LB } \\
\text { opt. }\end{array}$} & \multirow{2}{*}{$\begin{array}{l}\text { \# of } \\
\text { B\&B } \\
\text { opt. }\end{array}$} & \multirow{2}{*}{$\begin{array}{l}\text { Total time } \\
\text { (scs.) for all } \\
3 \text { instances }\end{array}$} \\
\hline & & & & conn. & odd & $\mathrm{K}-\mathrm{C}$ & $\mathrm{KC} 2$ & $\mathrm{HC}$ & $\mathrm{HC} 2$ & $\mathrm{nPB}$ & & & \\
\hline \multirow{3}{*}{16} & 0.3 & 3 & 4.0 & 13 & 26 & - & - & - & - & - & 3 & - & 0.11 \\
\hline & 0.5 & 3 & 4.3 & 13 & 28 & 2 & 2 & - & - & - & 3 & - & 0.11 \\
\hline & 0.7 & 3 & 4.0 & 14 & 30 & 19 & 6 & - & - & - & 3 & - & 0.21 \\
\hline \multirow{3}{*}{36} & 0.3 & 3 & 8.3 & 33 & 72 & 45 & 28 & - & - & 1 & 3 & - & 1.0 \\
\hline & 0.5 & 3 & 6.6 & 24 & 96 & 46 & 23 & 2 & 2 & - & 3 & - & 1.22 \\
\hline & 0.7 & 3 & 5.6 & 22 & 91 & 64 & 34 & 9 & 6 & - & 3 & - & 1.28 \\
\hline \multirow{3}{*}{64} & 0.3 & 3 & 12.0 & 57 & 137 & 51 & 22 & 3 & 2 & 4 & 3 & - & 2.75 \\
\hline & 0.5 & 3 & 9.0 & 35 & 226 & 102 & 43 & 14 & 9 & 1 & 3 & - & 9.19 \\
\hline & 0.7 & 3 & 5.0 & 21 & 151 & 80 & 26 & - & - & - & 3 & - & 2.97 \\
\hline \multirow{3}{*}{100} & 0.3 & 3 & 19.6 & 86 & 243 & 97 & 43 & 28 & 12 & 9 & 3 & - & 16.6 \\
\hline & 0.5 & 3 & 14.0 & 72 & 977 & 571 & 312 & 154 & 81 & 3 & 2 & 1 & 109 \\
\hline & 0.7 & 3 & 6.3 & 35 & 528 & 281 & 107 & 66 & 24 & - & 3 & - & 42.4 \\
\hline
\end{tabular}

Table 3: Computational results for Class 2 RPP random instances from Hertz et al. (1998). 


\begin{tabular}{|c|c|c|c|c|c|c|c|c|c|c|c|c|c|}
\hline \multirow[b]{2}{*}{$\mathrm{n}$} & \multirow[b]{2}{*}{ Prob. } & \multirow{2}{*}{$\begin{array}{l}\# \text { of } \\
\text { inst. }\end{array}$} & \multirow{2}{*}{$\begin{array}{l}\mathrm{R} \text {-sets } \\
\text { average }\end{array}$} & \multicolumn{7}{|c|}{ TOTAL constraints for all 3 instances } & \multirow{2}{*}{$\begin{array}{c}\text { \# of } \\
\text { LB } \\
\text { opt. }\end{array}$} & \multirow{2}{*}{$\begin{array}{l}\# \text { of } \\
\text { B\&B } \\
\text { opt. }\end{array}$} & \multirow{2}{*}{$\begin{array}{l}\text { Total time } \\
\text { (scs.) for all } \\
3 \text { instances }\end{array}$} \\
\hline & & & & conn. & odd & $\mathrm{K}-\mathrm{C}$ & $\mathrm{KC} 2$ & $\mathrm{HC}$ & $\mathrm{HC} 2$ & $\mathrm{nPB}$ & & & \\
\hline \multirow{3}{*}{16} & 0.3 & 30 & 3.0 & 10 & 24 & 6 & 2 & - & - & - & 3 & - & 0.09 \\
\hline & 0.5 & 3 & 3.6 & 13 & 26 & 4 & - & - & - & - & 3 & - & 0.19 \\
\hline & 0.7 & 3 & 4.6 & 16 & 34 & 8 & 2 & - & - & - & 3 & - & 0.22 \\
\hline \multirow{3}{*}{36} & 0.3 & 3 & 8.3 & 30 & 72 & 20 & 12 & - & - & - & 3 & - & 0.59 \\
\hline & 0.5 & 3 & 7.3 & 25 & 74 & 29 & 19 & - & - & - & 3 & - & 0.79 \\
\hline & 0.7 & 3 & 5.3 & 20 & 106 & 31 & 8 & - & - & - & 3 & - & 0.96 \\
\hline \multirow{3}{*}{64} & 0.3 & 3 & 12.3 & 45 & 122 & 49 & 34 & 3 & 2 & 1 & 3 & - & 2.04 \\
\hline & 0.5 & 3 & 10.3 & 43 & 273 & 106 & 57 & 13 & 6 & 6 & 3 & - & 8.61 \\
\hline & 0.7 & 3 & 6.6 & 23 & 192 & 69 & 40 & 7 & 4 & 3 & 3 & - & 3.46 \\
\hline \multirow{3}{*}{100} & 0.3 & 3 & 20.3 & 93 & 325 & 93 & 57 & 5 & 4 & 4 & 3 & - & 21.8 \\
\hline & 0.5 & 3 & 13.0 & 61 & 275 & 82 & 26 & 14 & 4 & 5 & 3 & - & 8.08 \\
\hline & 0.7 & 3 & 9.0 & 38 & 348 & 151 & 57 & 32 & 4 & - & 2 & 1 & 23.6 \\
\hline
\end{tabular}

Table 4: Computational results for Class 3 RPP random instances from Hertz et al. (1998).

\begin{tabular}{|c|c|c|c|c|c|c|c|c|c|c|c|c|c|c|}
\hline & \multirow{2}{*}{$\begin{array}{l}\text { R- } \\
\text { sets }\end{array}$} & \multirow[b]{2}{*}{ LB0 } & \multicolumn{7}{|c|}{ constraints } & \multirow[b]{2}{*}{ LB } & \multirow[b]{2}{*}{$B \& B$} & \multirow[b]{2}{*}{ UB } & \multirow{2}{*}{$\begin{array}{c}\text { time } \\
\text { (scs.) }\end{array}$} & \multirow{2}{*}{$\begin{array}{l}\# \text { o } \\
\text { iter. }\end{array}$} \\
\hline & & & conn. & odd & $\mathrm{K}-\mathrm{C}$ & $\mathrm{KC} 2$ & $\mathrm{HC}$ & $\mathrm{HC} 2$ & $\mathrm{nPB}$ & & & & & \\
\hline grp1 & 56 & 4183 & 84 & 127 & 53 & 16 & 8 & 3 & 9 & $5625^{*}$ & & & 42.1 & 14 \\
\hline grp2 & 52 & 3748 & 87 & 194 & 128 & 96 & 22 & 9 & 6 & $5569^{*}$ & & & 506 & 36 \\
\hline grp3 & 55 & 3937 & 90 & 125 & 31 & 16 & 4 & 4 & 1 & $5688^{*}$ & & & 65.4 & 13 \\
\hline grp4 & 48 & 2576 & 114 & 45 & 311 & 26 & 350 & 49 & - & 5119 & 5158 & 5186 & 1156 & 67 \\
\hline grp5 & 45 & 3620 & 68 & 115 & 26 & 16 & 12 & 6 & 2 & $5191^{*}$ & & & 119 & 13 \\
\hline grp6 & 10 & 2568 & 13 & 92 & - & - & - & - & - & $3442^{*}$ & & & 1.3 & 4 \\
\hline grp7 & 64 & 4525 & 103 & 113 & 52 & 27 & 18 & 4 & 2 & $6618^{*}$ & & & 110 & 14 \\
\hline grp8 & 47 & 5044 & 85 & 132 & 44 & 16 & 17 & 2 & 3 & $6814^{*}$ & & & 31.5 & 17 \\
\hline grp9 & 60 & 3329 & 93 & 92 & 17 & 12 & 15 & 8 & 3 & $4506^{*}$ & & & 207 & 15 \\
\hline grp10 & 48 & 2480 & 121 & - & 404 & 66 & 429 & 82 & - & 5029 & 5070 & 5122 & 2011 & 85 \\
\hline
\end{tabular}

Table 5: Computational results for 10 visually generated instances from the Albaida graph (116 vertices and 174 edges). 


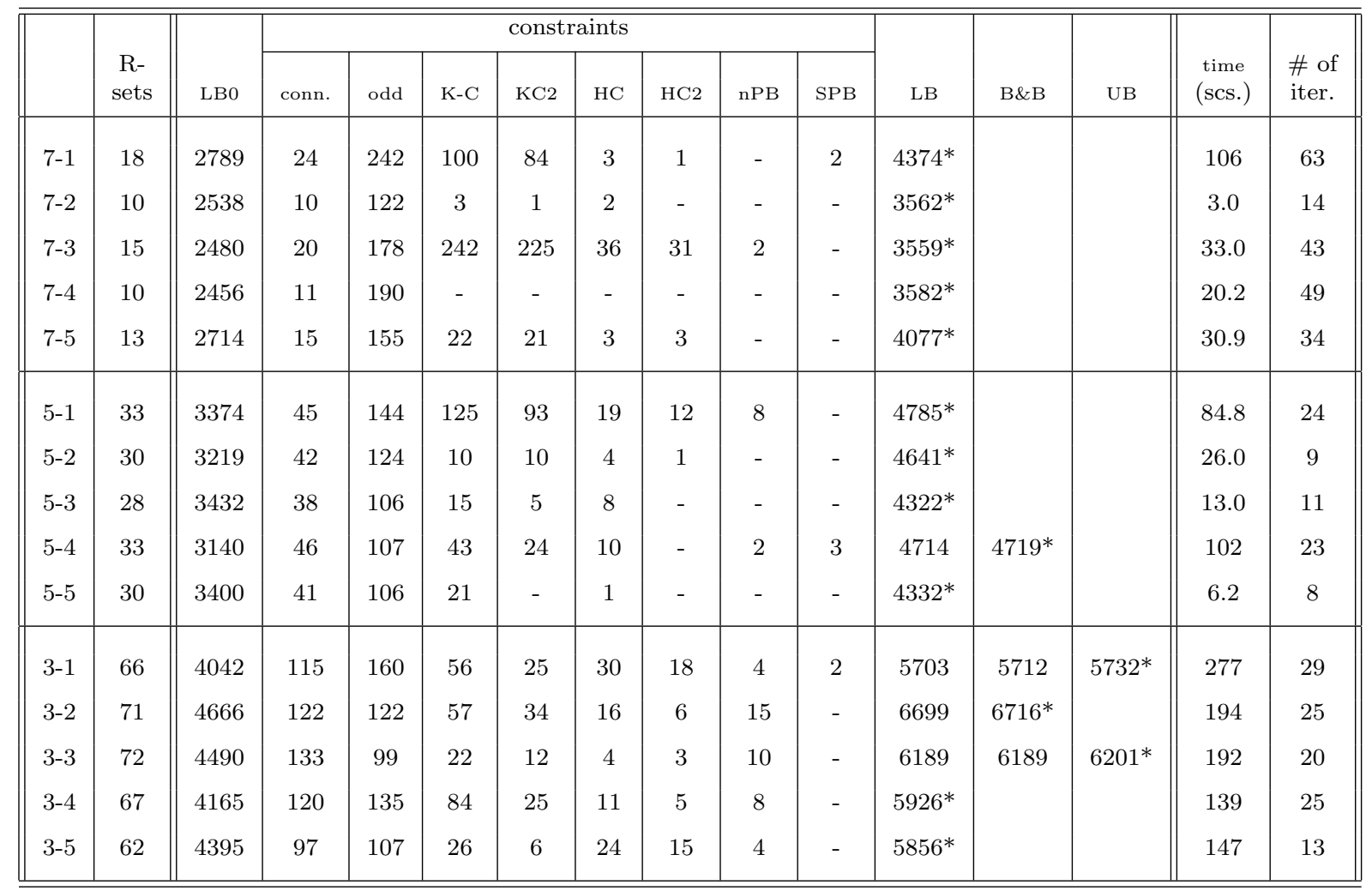

Table 6: Computational results for 15 randomly generated instances from the Albaida graph (116 vertices and 174 edges). 


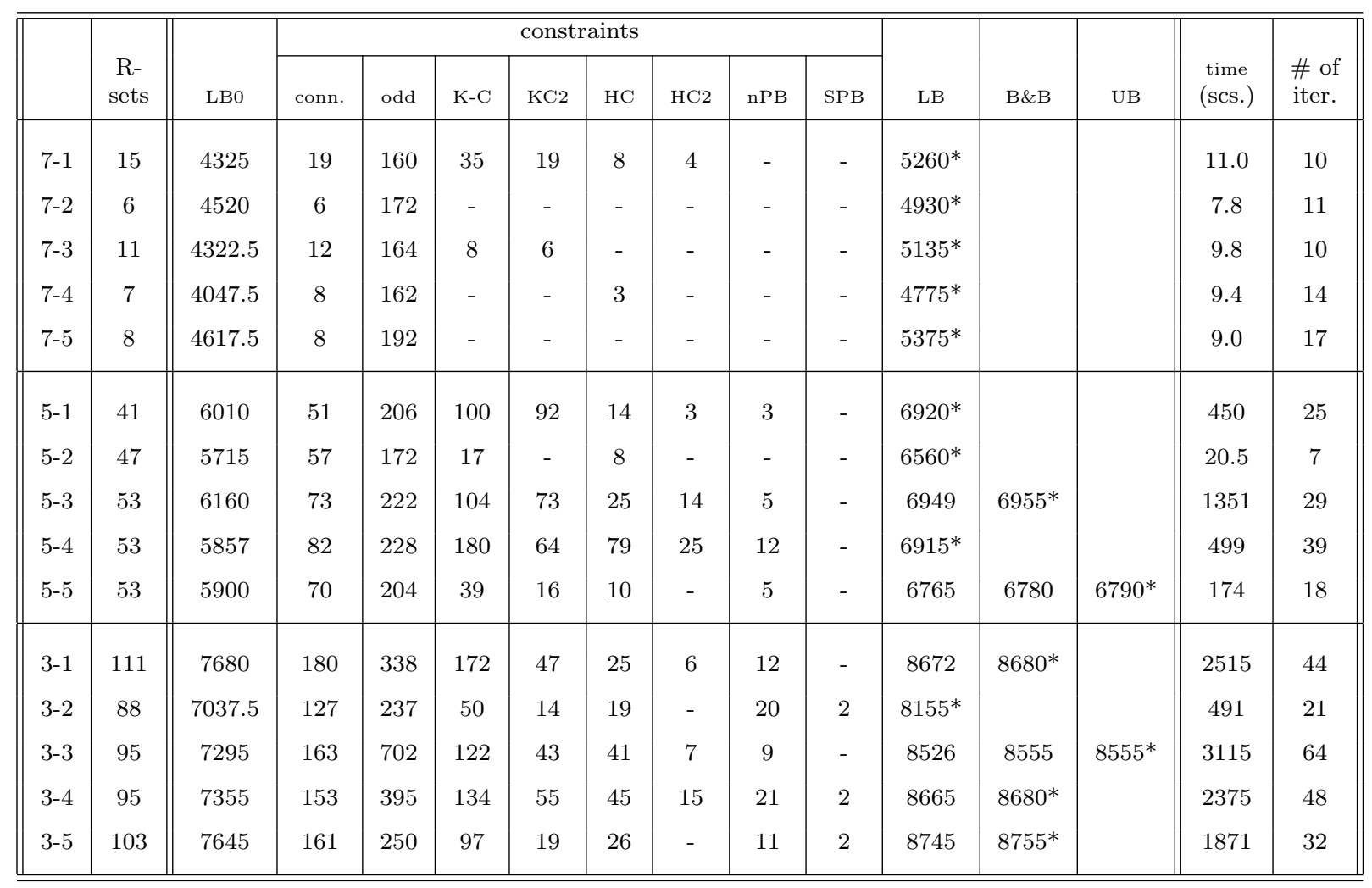

Table 7: Computational results for 15 randomly generated instances from the Madrigueras graph (196 vertices and 316 edges).

\begin{tabular}{||c||c|c|c|c|c|c|c||c|c||}
\hline \hline \multirow{2}{*}{} & \multirow{2}{*}{ LB0 } & \multicolumn{3}{|c|}{ constraints } & & & & & \\
\cline { 3 - 9 } & & conn. & nPB & SPB & LB & B\&B & UB & $\begin{array}{c}\text { time } \\
\text { (scs.) }\end{array}$ & $\begin{array}{c}\text { \# of } \\
\text { iter. }\end{array}$ \\
\hline \multirow{2}{*}{ kroA150g } & 21604.5 & 282 & 24 & - & 26489 & $26524^{*}$ & & 1045 & 23 \\
kroA200g & 23491.5 & 361 & 23 & - & $29368^{*}$ & & & 1142 & 15 \\
kroB150g & 20761.5 & 264 & 21 & - & $26130^{*}$ & & & 332 & 15 \\
kroB200g & 23888 & 342 & 8 & - & 29435 & $29437^{*}$ & & 775 & 13 \\
pr152g & 43232 & 360 & - & - & $73682^{*}$ & & & 74 & 11 \\
rat195g & 2097 & 369 & 48 & 12 & 2317 & $2323^{*}$ & & 15773 & 73 \\
ts225g & 115605 & 617 & 617 & - & 124591 & 125052 & $126643^{*}$ & 17976 & 265 \\
\hline
\end{tabular}

Table 8: Computational results for 7 GTSP instances generated from TSP instances in TSPLIB. 